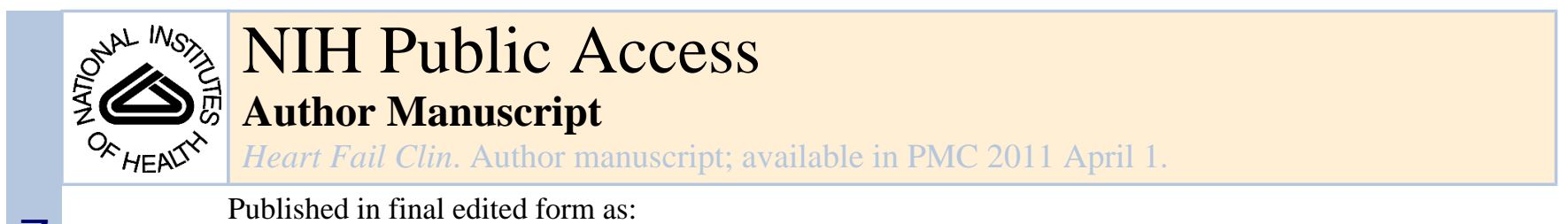

Published in final edited form as:

Heart Fail Clin. 2010 April ; 6(2): 231-238. doi:10.1016/j.hfc.2009.11.002.

\title{
Clinical screening and genetic testing
}

\author{
Rahul C. Deo, MD, PhD* and Calum A. MacRae, MBChB, PhD \\ Cardiology Division, Massachusetts General Hospital, Boston, MA
}

\begin{abstract}
Clinical screening lies at the heart of preventive medicine, since identification of a disease in its earliest form offers an opportunity to intervene and disrupt its expected deleterious course. In cardiovascular medicine, clinical screening is most effective in diseases such as hypercholesterolemia, where the disease in its earliest form may not have symptoms or signs but can be readily diagnosed with an inexpensive, non-invasive test. Other aspects of a disease like hypercholesterolemia also make a systematic screening program successful: it is relatively common, it has serious consequences such as myocardial infarction, and it is treatable, with the likelihood of adverse sequelae being reduced significantly by treatment. These and other criteria are used by groups, such as the US Preventive Task Force, to develop recommendations for screening programs (http://www.ahrq.gov/clinic/USpstfix.htm).
\end{abstract}

Genetic screening is a form of screening used for diseases with a significant heritable component. It involves searching for a one or more DNA variants in individuals believed to be at risk for a disease, where the DNA variant is believed to contribute to disease incidence or progression. Prior to comparing genetic and clinical screening, it would be helpful to review some aspects of the genetic basis of disease.

Genetic diseases lie along a continuum ranging from Mendelian disorders to complex diseases, which arise from the interaction of a number of genetic and environmental factors. Mendelian disorders typically arise from a mutation in a single gene and have a sufficiently dramatic effect that those who inherit the genetic mutation typically inherit the disease. The concept of penetrance captures the distinction between genetic variants contributing to Mendelian disorders and complex disease traits. Penetrance for a genetic mutation is defined as the proportion of individuals carrying a particular genetic mutation who also demonstrate the disease phenotype. The mutations that lead to Mendelian disorders have very high penetrances (approaching 100\%) while for most variants contributing to complex disease, the penetrance is quite low. This concept will have significant relevance when we discuss utility of genetic screening.

The concept of genetic architecture describes the number of genes contributing to a disease trait, the number of variants per gene, and the magnitude of effect that each variant has on development of the trait. Although Mendelian disorders usually arise from inheritance of a single genetic mutation, many different individual genes may, when mutated, lead to a common disease phenotype (genetic heterogeneity). Furthermore, for any gene, many different mutations may also lead to the same disease phenotype (allelic heterogeneity). Both genetic and allelic heterogeneity introduce complexity when one goes about designing a genetic screening program for cardiomyopathies. Furthermore, although the penetrance of a disorder

Corresponding Author: rdeo@partners.org.

Publisher's Disclaimer: This is a PDF file of an unedited manuscript that has been accepted for publication. As a service to our customers we are providing this early version of the manuscript. The manuscript will undergo copyediting, typesetting, and review of the resulting proof before it is published in its final citable form. Please note that during the production process errors may be discovered which could affect the content, and all legal disclaimers that apply to the journal pertain. 
may be high, the exact manifestation of disease may vary from individual to individual, despite inheriting the same mutation (variable expressivity). A final level of complexity arises from the fact that multiple distinct diseases may share a common "low-resolution" phenotype, but in fact have a different pathologic basis (termed phenocopies), with potentially different disease course and treatment.

Genetic screening differs from clinical screening in several regards. Rather than serve as a way of diagnosing disease in asymptomatic individuals, the identification of a risk variant in an individual can give the probability of disease risk in individuals who may not yet have disease. Acting on this information may not only allow prevention of disease progression, but also the prevention of disease incidence, the "holy grail" of medicine. A second difference is that discovering that individuals with subclinical disease have a genetic risk variant may provide insight into the biological basis of disease for that individual. For clinically heterogeneous diseases, such as atherosclerosis or hypertension, understanding the driving pathophysiologic progress may allow targeted therapy that may surpass the efficacy of the "one treatment fits all" approach commonly used. Moreover, with some limitations, knowledge of the causal process may permit a more accurate prognosis of catastrophic outcomes such as sudden cardiac death or stroke and allow the focused implementation of screening or preventive therapeutic procedures that may be too costly or risky for the general population, but have high likelihood of benefit for a limited number of high risk individuals.

When should genetic screening used? An example may help illustrate the approach we use for potentially heritable disorders. Consider an individual with a disease that does not appear to be arising from any known environmental cause - in genetic studies, this individual is called the proband. An initial step should be to establish whether the disease is familial, as this has relevance to pursuing a genetic diagnosis for the individual, and on managing risk within family members. In addressing familiality, we must construct a careful family pedigree, asking about the health and manner of death of every relative. We need to be careful to distinguish two apparently similar situations, with considerably different ramifications: one where detailed pedigree information is available and no disease is apparent, versus another where there does not appear to be any other relative with the disorder but inadequate family history is obtained. Only in the former case could we conclude that the disease is not familial, and either sporadic or attributable to environmental factors. If the proband has multiple relatives with the disorder, we would consider it to be familial, and consider genetic screening.

The next considerations are related to our likelihood of identifying a causal variant in the proband. If the genetic architecture of the disease is such that there are a relatively small number of genes (low genetic heterogeneity) involved and there are causal genetic variants of moderate to high penetrance, genetic screening can be useful. Because many Mendelian disorders show significant allelic heterogeneity, screening for a single mutation tends to be unsuccessful, and sequencing of portions of the gene (exons, splice junctions) tend to be required to find likely causal variants. Several limitations exist with genetic testing of a single proband. Sequencing errors can occur, resulting in false positives and false negative results. Even with careful sequencing, a variant may be found in one of the candidate genes but not actually be causal for the disease. To establish a sequence variant as a potential mutation would require that it have the potential to have a deleterious effect (missense or nonsense) and lie within a protein domain previously attributed functional significance. A mutation that is falsely assigned causality and used for genetic screening in family members would lead to both false reassurance and false alarm, as the inheritance of the variant would have no bearing on the likelihood of developing the disease. This situation may be ameliorated if a large number of family members are available for genetic testing, as co-segregation of mutation with disease can be used to infer causality. 
How useful would the identification of a genetic variant be? Because of the bewildering genetic and allelic heterogeneity of most Mendelian disorders, the individualized prognostication and treatment that was once hoped to follow genetic diagnoses has not materialized. We simply do not have enough prognostic information for individual mutations to provide mutation-specific predictions with any accuracy. As a result, the current utility of identifying a causal mutation in a proband is almost exclusively limited to facilitating screening of family members. In particular, with the help of genetic screening, we can help identifying affected individuals at a preclinical phase, or those with ambiguous clinical screening results.

A "cascade screening" approach allows an efficient method of evaluating which family members carry the causal allele. Once a genetic diagnosis of the proband is made, all of the first-degree relatives of the proband are screened. We can limit further genetic screening to first-degree relatives of the proband's affect first-degree relatives. This process continues until no further affected individual is identified. Genetic diagnosis allows a considerable degree of reassurance to family members who are genotype-negative, as they no longer need clinical surveillance, and need not worry that disease will be passed on to their progeny. Conversely, a positive diagnosis in a clinically unaffected individual may lead to initiation of more frequent surveillance, avoidance of high risk behavior, implementation of preventive treatment, and potentially it may affect reproductive choices. Of course, as discussed above, the success of such an approach depends fully on confidence that the mutation used for screening is actually causal.

If a causal genetic variant cannot be definitively established for the proband, clinical screening should then be considered, as it can be useful in many of the same ways as genetic screening. Cascade screening, described above, cannot work for clinical screening because of incomplete age-dependent penetrance, which may lead to premature termination of screening if any individual failed to display features of the disease. Thus all relatives of the proband should be screened. The age of screening typically depends on the range of age of onset for the disease.

We can apply the above considerations to any disease with a heritable component. Below, we will address the screening approaches to dilated cardiomyopathy (DCM), hypertrophic cardiomyopathy (HCM), arrhythmogenic right ventricular dysplasia (ARVD) and restrictive cardiomyopathy (RCM), highlighting how the known genetic architecture of the trait guides a genetic screening approach and how clinical characteristics of the disease influence a clinical screening approach.

\section{Hypertrophic Cardiomyopathy}

The genetic architecture of hypertrophic cardiomyopathy (Table 1) makes it amenable to genetic diagnosis. HCM appears to be familial in approximately $50 \%$ of cases and the inheritance pattern in documented cases is almost always autosomal dominant with high penetrance [1,2]. There are 12 known genes responsible for this disorder (not including several phenotypic mimics [3]), and mutations in the exons or splice junctions of one of eight sarcomeric genes explain approximately $50-60 \%$ of cases [4]. There are various commercial tests for genetic screening. The Center for Genetics and Genomics at Harvard Medical School offers a $\$ 3000$ screening test for 106 exons and splice sites of 5 sarcomeric genes (MYH7, MYBPC3, TNNT2, TNNI3, TPM1), and an additional \$1150 screening test for 19 exons and splice sites in 3 other genes (ACTC, MYL2, MYL3) (http://www.hpcgg.org/LMM/comment/HCM\%20Info\%20Sheet.htm). Similarly, PGxHealth offers sequencing of all 113 exons in 9 genes (above 8 genes as well as TNNC1, http://www.pgxhealth.com/genetictests/familion/). Despite the availability of commercial sequencing services, the prevalence of HCM, which is $0.2-0.5 \%$ of the general population, is too low to justify screening of the general population. 
The allelic heterogeneity of HCM, which includes over 400 causal mutations, makes individualization of treatment and prognosis on the basis of genetics implausible. It is highly unlikely that for any mutation, adequate samples will ever be assembled for a reliable estimate of risk. Furthermore, incomplete penetrance and variable expressivity within families further erode confidence in the predictive utility of mutations. Attempts to prognosticate on the basis of genetic mutations have been difficult to replicate, and designations of mutations as "benign" or "malignant" are often based on observational studies in small numbers of families [5]. It is of course expected that some mutations will have a more deleterious impact on protein function than others - but to extrapolate the clinical impact of a single mutation from a small number of individuals to others with different genetic and environmental backgrounds should be undertaken only with caution.

Thus, at present, a genetic diagnosis is most useful for screening relatives of the proband, with a cascade-type approach, as described above. If a genetic diagnosis is not pursued or made, clinical screening can be carried out using ECG and echocardiography. Echocardiography has greater specificity, although ECG findings may precede changes in LV thickness. ECG abnormalities, even in the absence of LVH, on echocardiography are suggestive for affected status, especially given the high pre-test probability of disease in first-degree relatives.

There is uncertainty as to the age at which clinical screening should be initiated or terminated. Given the concern for sudden death in child athletes, an early diagnosis of HCM in children has clear relevance to mitigating risk. Furthermore, as multiple HCM variants can show clinical onset late in life [6], it is unclear if screening can be stopped confidently at any age. Maron et al, have recommended optional screening for age $<12$ years (unless family history of premature sudden death, symptoms, or plan to pursue strenuous sporting activity), 12-18 month screening intervals for children 12 to 18-21 years old, and screening every 5 years for ages >18-21 years [6]. Although representing a rational approach, there have been no efforts yet to validate this strategy in any large population for cost effectiveness or influence on morbidity or mortality.

A pre-clinical diagnosis of HCM, either through genetic or clinical screening, leaves us with the opportunity to make clinical decisions prior to disease onset. Unfortunately, there are no clear options for treatment to alter the course of disease. Sudden death in HCM is certainly the most dreaded sequelum, and the possibility exists of implanting a defibrillator for primary prevention. Unfortunately, we are not confident which HCM patients will benefit most from this therapy. At present, just as with ischemic cardiomyopathy, our best predictor of sudden death in HCM patients is a personal history of cardiac arrest; 59\% of individuals with one episode of cardiac arrest have a second one within 5 years [7]. However, in the absence of a prior cardiac arrest, the criteria for risk prediction become less clear. A personal history of unexplained syncope or a family history of sudden cardiac death [8] has modest additional predictive utility. The caveats describe above that apply to establishing familiality also apply to establishing a family history for sudden death - one must be concerned if there are simply not enough family members on which to base a negative conclusion. In other studies, features such as LV thickness, non-sustained VT and abnormal BP response in exercise have been implicated as potential predictors of sudden death, but these studies did not account for familiality, and no formal validation of any of these prognostic models has occurred.

\section{Dilated Cardiomyopathy}

DCM is considerably more complex than HCM, both in terms of genetic architecture and known contributing environmental factors. Coronary artery disease, nutritional deficiency, viral infection and toxins such as alcohol all can cause DCM, though familial predisposition may continue to play a role in many of these cases. The prevalence of DCM may be as high as 1 in 2500 adults [9]. Given that the histologic findings of DCM are non-specific with myocyte 
loss and interstitial fibrosis, a diagnosis of idiopathic DCM requires an extensive work-up to exclude other causes, some of which may prove to be reversible [10].

Over 20 genes have been implicated in the pathogenesis of DCM, with autosomal dominant, recessive and X-linked patterns of inheritance [11] (Table 1). Penetrance is often low, and expressivity varies considerably from individual to individual. Dilated cardiomyopathy can be syndromic, with other accompanying systemic abnormalities such as the skeletal muscle dystrophies and retinal disease [12]. Given the fact that mutations in DCM are distributed widely over a large number of different potential causal genes, there is usually too low a likelihood of success to recommend genetic sequencing or genetic screening. Interestingly, it is the associated cardiac and non-cardiac findings that can help narrow the diagnosis. For example, in one small study, if AV block accompanied DCM, there was a mutation found in the lamin $\mathrm{A} / \mathrm{C}$ gene in $1 / 3$ of cases [13].

As with all cardiomyopathies, it is challenging to predict risk for particular mutations. One exception may be a tendency for DCM caused by lamin A/C mutations to demonstrate a high rate of malignant arrhythmias in patients with conduction abnormalities [14]. This finding has not yet been replicated.

Although the complexity of DCM precludes genetic screening, clinical screening can often be very useful. Moreover, an early diagnosis in asymptomatic family members of the proband allows the initiation of potentially disease-modifying agents such as ACE-inhibitors (see below). Clinical screening is performed by echocardiography and ECG. Individuals with ECG abnormalities or mild echocardiographic abnormalities (mildly depressed systolic ejection fraction or mild LV enlargement) should be followed with more frequent screening. As with $\mathrm{HCM}$, there are no explicit evidence-based guidelines for screening, although it would be reasonable to begin in childhood and continue at periodic intervals into late adulthood. For every affected individual, care must be taken to exclude age-appropriate, potentially reversible causes (e.g. tachyarrhythmia, CAD, alcohol, etc.,) as these may contribute to disease even in the context of an inherited tendency [15]

The forbidding genetic and phenotypic heterogeneity of DCM makes genotype-based treatment unlikely. Clinical guidelines recommend ACE-I and beta blocker use for all dilated cardiomyopathies, independent of cause [16] and AICD implantation in symptomatic individuals with severe LV dysfunction. It is unclear if early initiation of ACE-I or beta blockers mitigates the disease course in individuals with mild echocardiographic abnormalities, or exclusively ECG abnormalities. We tend to favor the use of ACE-I in such cases, given the efficacy in asymptomatic LV dysfunction of all types.

\section{Arrhythmogenic Right Ventricular Dysplasia}

Arrhythmogenic right ventricular dysplasia (ARVD) is a genetically heterogeneous disorder, with twelve current genetic loci (ARVD1-12) identified through linkage studies [17] (Table 1). Causal genes corresponding to eight of these loci have been found, with five encoding desmosomal proteins. The prevalence of ARVD is unknown but has been estimated at 1:1000 to 1:5000 individuals [18]. ARVD is familial in nearly 50\% of cases [19], and inheritance is usually autosomal dominant, with variable expressivity and incomplete penetrance.

The routine diagnostic workup of a patient suspected with ARVD includes ECG, Holter, signalaveraged ECG, echocardiogram and potentially cardiac magnetic resonance [20]. If the clinical and family history and these initial studies raise a high suspicion for ARVD, endomyocardial biopsy is often performed for confirmation and EP study may be performed to exclude benign right-ventricular outflow-tract tachycardia. The above diagnostic tests have been incorporated into task force criteria (TFC) for the diagnosis of ARVD [21] (see table \#). 
The frequency distribution of causal genes appears to vary with geographic region, although a large percentage (up to $43 \%$ ) of cases can be explained by mutations in the plakophilin 2 (PKP2) gene [22,23]. As with HCM, allelic heterogeneity is present, with over 50 PKP2 mutations currently known [17]. The penetrance of ARVD mutations appears lower than HCM, potentially due to the insensitivity of the TFC $[19,24]$. Sequencing of the most commonly mutated genes may be useful in identifying family members of the proband who require longterm clinical followup, especially since correct identification of affected individuals may be useful in prevention of sudden cardiac death. Towards those ends, the Center for Genetics and Genomics at Harvard Medical School also offers sequencing of 69 exons and splice sites for the four most common genes mutated in ARVD (PKP2, desmoplakin, desmoglein 2, and demoscollin 2), for a cost of $\$ 3000$. It is unclear what percentage of probands will be identified through this assay. Once a mutation is found, additional family members can be screened at a cost of \$250 each.

As with HCM, the relevance of genetic diagnosis to prognostication or individualization of therapy is limited by the fact that most mutations identified to date are rare and "private" to individual families [25]. Futhermore, given incomplete penetrance and variable expressivity within families, it is unclear to what extent we can extrapolate the sudden death risk from one family with a given mutation to another, even if they share the same mutation. Given the wide range of effects that mutations can have on protein function, ranging from little to no change in activity to severe dominant negative action, it is highly unlikely that we will be able to define a common risk profile for all mutations of a single gene, such has been proposed for desmoplakin [26] and PKP2 [27,28].

If a genetic diagnosis cannot be made for the proband, clinical screening of family members would occur initially by ECG and echocardiogram. Abnormalities on either of these would result in further testing as described above, with a low threshold for declaring a positive diagnosis even if formal TFC are not met, given the high prior probability of disease. As a result of early onset of disease and the potential hazards of exercise on disease progression, screening for ARVD should begin in childhood. For genetic screening, all first-degree relatives should be screened initially, with cascade screening, as described above. If clinical screening is being performed, individuals who appear "negative" for disease should continue to be screened at some regular interval [29]. The late appearance of ARVD in some individuals [30] requires that screening should continue throughout adult life. Nava et al used a systematic (uncontrolled) screening and prevention approach in 37 families with ARVD, and demonstrated that frequent screening, initiation of anti-arrhythmics as needed, and avoidance of exercise led to very low mortality among affected individuals [29]. A similar approach could be extrapolated to asymptomatic individuals harboring a potential ARVD mutation.

ICD implantation in patients with the diagnosis of ARVD remains an area of uncertainty. Piccini et al recommend ICDs for all ARVD patients meeting TFC [31], given the high risk of ventricular tachycardia, even in patients with no prior history of syncope or cardiac arrest. As with HCM, attempts have been made to identify high-risk diagnostic features with high positive predictive value for sudden cardiac death, such as RV dysfunction, LV dysfunction and recurrent VT $[32,33]$. A consensus on criteria for ICD implantation has yet to appear.

\section{Restrictive Cardiomyopathy}

Restrictive cardiomyopathies (RCM) demonstrate several rare hereditary variants, including familial idiopathic restrictive cardiomyopathy, and hereditary amyloidosis. Familial idiopathic RCM is extremely rare, with reports only in small case series [34,35]. No gene has yet been identified. Furthermore, in some families with HCM, individual members can show a pattern of restrictive filling with little or no LV hypertropy [36,37]. In a systematic analysis of 1226 
relatives of HCM probands, this "restrictive phenotype" of HCM was seen in 1.5\% of individuals, and the diagnosis was accompanied by a high rate of dypsnea and mortality.

Hereditary amyloidosis represents a more common form of heritable RCM and typically involves a genetic defect in the transthyretin (TTR) protein or Apo AI protein leading to misfolded proteins and infiltration of the myocardium with amyloid fibrils. RCM shows allelic heterogeneity, with over TTR mutations identified to date [38]. The inheritance pattern is usually autosomal dominant.

An RCM patient with evidence of a familial inheritance pattern should undergo right heart catheterization with right ventricular biopsy to evaluate for infiltrative disease. If amyloid deposits are found, hereditary amyloidosis should be presumed, and TTR sequencing performed to identify the causal variant. The identified variant can be used for genetic screening. If no amyloid deposits are seen, one should suspect an idiopathic variant, and clinical screening of family members by echocardiography and ECG should be performed.

Unfortunately, none of the treatment measures for RCM have been shown to impact mortality. Loop diuretics, calcium channel blockers, beta-blockers, and ACE-inhibitors are commonly used for relief of symptoms.

\section{Conclusions}

General principles of genetic disease architecture can guide screening and diagnostic approaches for all of the cardiomyopathies, and in fact, for all inherited diseases. At present, the primary benefit of identifying a causal mutation in a proband is to facilitate screening in family members. A preclinical diagnosis achieved through screening programs can allow initiation of further monitoring programs for disease development, avoidance of high-risk behaviors, and potential implementation of disease-mitigating therapies. Although there is considerable incentive to offer genotype-based forecasting for patients, allelic and genetic heterogeneity and variable expressivity have rendered such individualization of care highly unlikely. Our ultimate desire for tailored prognostication and therapy is likely only to be realized when we can generate phenotypic profiles that can integrate individual genotypic and environmental information and yet be common enough to allow accuracy in prediction and classification.

\section{References}

1. Ho CY, Seidman CE. A contemporary approach to hypertrophic cardiomyopathy. Circulation 2006;113:e858-62. [PubMed: 16785342]

2. Ashrafian $\mathrm{H}$, Watkins $\mathrm{H}$. Reviews of translational medicine and genomics in cardiovascular disease: new disease taxonomy and therapeutic implications cardiomyopathies: therapeutics based on molecular phenotype. J Am Coll Cardiol 2007;49:1251-64. [PubMed: 17394955]

3. Arad M, Maron BJ, Gorham JM, et al. Glycogen storage diseases presenting as hypertrophic cardiomyopathy. N Engl J Med 2005;352:362-72. [PubMed: 15673802]

4. Richard P, Charron P, Carrier L, et al. Hypertrophic cardiomyopathy: distribution of disease genes, spectrum of mutations, and implications for a molecular diagnosis strategy. Circulation 2003;107:2227-32. [PubMed: 12707239]

5. Ackerman MJ, VanDriest SL, Ommen SR, et al. Prevalence and age-dependence of malignant mutations in the beta-myosin heavy chain and troponin $\mathrm{T}$ genes in hypertrophic cardiomyopathy: a comprehensive outpatient perspective. J Am Coll Cardiol 2002;39:2042-8. [PubMed: 12084606]

6. Maron BJ, Seidman JG, Seidman CE. Proposal for contemporary screening strategies in families with hypertrophic cardiomyopathy. J Am Coll Cardiol 2004;44:2125-32. [PubMed: 15582308] 
7. Elliott PM, Sharma S, Varnava A, et al. Survival after cardiac arrest or sustained ventricular tachycardia in patients with hypertrophic cardiomyopathy. J Am Coll Cardiol 1999;33:1596-601. [PubMed: 10334430]

8. Elliott PM, Poloniecki J, Dickie S, et al. Sudden death in hypertrophic cardiomyopathy: identification of high risk patients. J Am Coll Cardiol 2000;36:2212-8. [PubMed: 11127463]

9. Codd MB, Sugrue DD, Gersh BJ, et al. Epidemiology of idiopathic dilated and hypertrophic cardiomyopathy. A population-based study in Olmsted County, Minnesota, 1975-1984. Circulation 1989;80:564-72. [PubMed: 2766509]

10. Mestroni L, Maisch B, McKenna WJ, et al. Guidelines for the study of familial dilated cardiomyopathies. Collaborative Research Group of the European Human and Capital Mobility Project on Familial Dilated Cardiomyopathy. Eur Heart J 1999;20:93-102. [PubMed: 10099905]

11. Taylor MR, Slavov D, Ku L, et al. Prevalence of desmin mutations in dilated cardiomyopathy. Circulation 2007;115:1244-51. [PubMed: 17325244]

12. Marshall JD, Hinman EG, Collin GB, et al. Spectrum of ALMS1 variants and evaluation of genotypephenotype correlations in Alstrom syndrome. Hum Mutat. 2007

13. Arbustini E, Pilotto A, Repetto A, et al. Autosomal dominant dilated cardiomyopathy with atrioventricular block: a lamin A/C defect-related disease. J Am Coll Cardiol 2002;39:981-90. [PubMed: 11897440]

14. Meune C, Van Berlo JH, Anselme F, et al. Primary prevention of sudden death in patients with lamin A/C gene mutations. N Engl J Med 2006;354:209-10. [PubMed: 16407522]

15. Mahon NG, Murphy RT, MacRae CA, et al. Echocardiographic evaluation in asymptomatic relatives of patients with dilated cardiomyopathy reveals preclinical disease. Ann Intern Med 2005;143:10815. [PubMed: 16027452]

16. Hunt SA. ACC/AHA 2005 guideline update for the diagnosis and management of chronic heart failure in the adult: a report of the American College of Cardiology/American Heart Association Task Force on Practice Guidelines (Writing Committee to Update the 2001 Guidelines for the Evaluation and Management of Heart Failure). J Am Coll Cardiol 2005;46:e1-82. [PubMed: 16168273]

17. van Tintelen JP, Hofstra RM, Wiesfeld AC, et al. Molecular genetics of arrhythmogenic right ventricular cardiomyopathy: emerging horizon? Curr Opin Cardiol 2007;22:185-92. [PubMed: 17413274]

18. Peters S, Trummel M, Meyners W. Prevalence of right ventricular dysplasia-cardiomyopathy in a non-referral hospital. Int J Cardiol 2004;97:499-501. [PubMed: 15561339]

19. Hamid MS, Norman M, Quraishi A, et al. Prospective evaluation of relatives for familial arrhythmogenic right ventricular cardiomyopathy/dysplasia reveals a need to broaden diagnostic criteria. J Am Coll Cardiol 2002;40:1445-50. [PubMed: 12392835]

20. Calkins H. Arrhythmogenic right-ventricular dysplasia/cardiomyopathy. Curr Opin Cardiol 2006;21:55-63. [PubMed: 16355031]

21. McKenna WJ, Thiene G, Nava A, et al. Diagnosis of arrhythmogenic right ventricular dysplasia/ cardiomyopathy. Task Force of the Working Group Myocardial and Pericardial Disease of the European Society of Cardiology and of the Scientific Council on Cardiomyopathies of the International Society and Federation of Cardiology. Br Heart J 1994;71:215-8. [PubMed: 8142187]

22. Dalal D, James C, Devanagondi R, et al. Penetrance of mutations in plakophilin-2 among families with arrhythmogenic right ventricular dysplasia/cardiomyopathy. J Am Coll Cardiol 2006;48:141624. [PubMed: 17010805]

23. van Tintelen JP, Entius MM, Bhuiyan ZA, et al. Plakophilin-2 mutations are the major determinant of familial arrhythmogenic right ventricular dysplasia/cardiomyopathy. Circulation 2006;113:16508. [PubMed: 16567567]

24. Kies P, Bootsma M, Bax JJ, et al. Serial reevaluation for ARVD/C is indicated in patients presenting with left bundle branch block ventricular tachycardia and minor ECG abnormalities. J Cardiovasc Electrophysiol 2006;17:586-93. [PubMed: 16836703]

25. Wichter T, Breithardt G. Implantable cardioverter-defibrillator therapy in arrhythmogenic right ventricular cardiomyopathy: a role for genotyping in decision-making? J Am Coll Cardiol 2005;45:409-11. [PubMed: 15680720] 
26. Bauce B, Basso C, Rampazzo A, et al. Clinical profile of four families with arrhythmogenic right ventricular cardiomyopathy caused by dominant desmoplakin mutations. Eur Heart J 2005;26:166675. [PubMed: 15941723]

27. Dalal D, Molin LH, Piccini J, et al. Clinical features of arrhythmogenic right ventricular dysplasia/ cardiomyopathy associated with mutations in plakophilin-2. Circulation 2006;113:1641-9. [PubMed: 16549640]

28. Syrris P, Ward D, Asimaki A, et al. Clinical expression of plakophilin-2 mutations in familial arrhythmogenic right ventricular cardiomyopathy. Circulation 2006;113:356-64. [PubMed: 16415378]

29. Nava A, Bauce B, Basso C, et al. Clinical profile and long-term follow-up of 37 families with arrhythmogenic right ventricular cardiomyopathy. J Am Coll Cardiol 2000;36:2226-33. [PubMed: 11127465]

30. Frigo G, Bauce B, Basso C, et al. Late-onset arrhythmogenic right ventricular cardiomyopathy. J Cardiovasc Med (Hagerstown) 2006;7:74-6. [PubMed: 16645365]

31. Piccini JP, Dalal D, Roguin A, et al. Predictors of appropriate implantable defibrillator therapies in patients with arrhythmogenic right ventricular dysplasia. Heart Rhythm 2005;2:1188-94. [PubMed: 16253908]

32. Roguin A, Bomma CS, Nasir K, et al. Implantable cardioverter-defibrillators in patients with arrhythmogenic right ventricular dysplasia/cardiomyopathy. J Am Coll Cardiol 2004;43:1843-52. [PubMed: 15145110]

33. Lemola K, Brunckhorst C, Helfenstein U, et al. Predictors of adverse outcome in patients with arrhythmogenic right ventricular dysplasia/cardiomyopathy: long term experience of a tertiary care centre. Heart 2005;91:1167-72. [PubMed: 16103549]

34. Fitzpatrick AP, Shapiro LM, Rickards AF, et al. Familial restrictive cardiomyopathy with atrioventricular block and skeletal myopathy. Br Heart J 1990;63:114-8. [PubMed: 2317404]

35. Angelini A, Calzolari V, Thiene G, et al. Morphologic spectrum of primary restrictive cardiomyopathy. Am J Cardiol 1997;80:1046-50. [PubMed: 9352976]

36. Kubo T, Gimeno JR, Bahl A, et al. Prevalence, clinical significance, and genetic basis of hypertrophic cardiomyopathy with restrictive phenotype. J Am Coll Cardiol 2007;49:2419-26. [PubMed: 17599605]

37. Mogensen J, Kubo T, Duque M, et al. Idiopathic restrictive cardiomyopathy is part of the clinical expression of cardiac troponin I mutations. J Clin Invest 2003;111:209-16. [PubMed: 12531876]

38. Hughes SE, McKenna WJ. New insights into the pathology of inherited cardiomyopathy. Heart 2005;91:257-64. [PubMed: 15657260] 


\section{Table 1}

Genetic Architecture of Hypertrophic and Dilated Cardiomyopathies and Arrhythmogenic Right Ventricular Dysplasia

\begin{tabular}{|c|c|c|c|}
\hline & HCM & DCM & ARVD \\
\hline Prevalence & $1 / 500$ & $1 / 2500$ & $1 / 1000-1 / 5000$ \\
\hline No. known causal genes & 12 & 20 & 7 \\
\hline No. known variants & $>400$ & $>50$ & $>70$ \\
\hline Familiality & $50 \%$ & $35 \%$ & $30-50 \%$ \\
\hline Predominant patterns of inheritance & Autosomal dominant & $\begin{array}{c}\text { Autosomal dominant, Autosomal recessive, } \\
\text { X-linked }\end{array}$ & $\begin{array}{c}\text { Autosomal dominant, } \\
\text { Autosomal recessive }\end{array}$ \\
\hline Potential Preventive Treatment & AICD & ACE-I, beta-blocker, AICD & Avoidance of exercise, AICD \\
\hline
\end{tabular}

